7-1983

\title{
Highly Excited States of a Hydrogen Atom in a Strong Magnetic Field
}

John B. Delos

William \& Mary, jbdelos@wm.edu

Stephen Knudson

D. W. Knudson

Follow this and additional works at: https://scholarworks.wm.edu/aspubs

Part of the Physics Commons

\section{Recommended Citation}

Delos, John B.; Knudson, Stephen; and Knudson, D. W., Highly Excited States of a Hydrogen Atom in a Strong Magnetic Field (1983). Physical Review A, 28(1), 7-21.

https://doi.org/10.1103/PhysRevA.28.7

This Article is brought to you for free and open access by the Arts and Sciences at W\&M ScholarWorks. It has been accepted for inclusion in Arts \& Sciences Articles by an authorized administrator of W\&M ScholarWorks. For more information, please contact scholarworks@wm.edu. 


\title{
Highly excited states of a hydrogen atom in a strong magnetic field
}

\author{
J. B. Delos \\ Physics Department, College of William and Mary, Williamsburg, Virginia 23185 \\ S. K. Knudson \\ Chemistry Department, College of William and Mary, Williamsburg, Virginia 23185 \\ D. W. Noid \\ Oak Ridge National Laboratory, Oak Ridge, Tennessee 37830 \\ and Department of Chemistry, University of Tennessee, Knoxville, Tennessee 37916 \\ (Received 8 October 1982)
}

\begin{abstract}
Classical trajectories and semiclassical energy eigenvalues are calculated for an atomic electron in a high Rydberg state in an external magnetic field. With the use of perturbation theory, a classical trajectory is described as a Kepler ellipse with orbital parameters evolving slowly with time. As they evolve, the ellipse rocks, tilts, and flips in space, but the length of its major axis remains approximately constant. Exact numerical calculations verify that perturbation theory is quite accurate for the cases considered (principal quantum number $\simeq 30$, magnetic field $\leq 6 \mathrm{~T}$ ). Action variables are calculated from perturbation theory and from exact trajectories, and semiclassical eigenvalues are obtained by quantization of action. Excellent agreement is found with observations.
\end{abstract}

\section{INTRODUCTION}

Since the beginning of this century, it has been known that when an atom is placed in a magnetic field, the magnetic moments associated with orbital and spin angular momenta are coupled to the field, splitting individual atomic spectral lines into several components. Very detailed quantitative descriptions of these effects were obtained, ${ }^{1,2}$ and the phenomena are now so familiar that it might seem that there is nothing more to be learned about isolated one-electron atoms in magnetic fields. However, these familiar effects appear only when the magnetic field is very weak compared to the electrostatic field. Recently it has become clear that as the magnetic field is increased, an atom will display a very rich diversity of behavior. In this paper, we explore some of the interesting phenomena that appear if the magnetic field is not so weak. ${ }^{3}$

Let us begin by sorting out the ranges of magnetic field strength in which different types of behavior can occur. The Hamiltonian for an electron in a magnetic field contains $(\overrightarrow{\mathrm{p}}+e \overrightarrow{\mathscr{A}} / c)^{2}$, where $\overrightarrow{\mathscr{A}}$ is the vector potential $(\overrightarrow{\mathbf{B}}=\vec{\nabla} \times \overrightarrow{\mathscr{A}})$; there are therefore two terms, one linear and one quadratic in $B$. Let us define the "linear" regime as that range of field strengths and quantum states in which the linear term is significant but the quadratic term is negligible. Phenomena that occur here include the "normal" and "anomalous" Zeeman effects and the PaschenBack effect, all of which have been extensively studied.

We define the "quadratic regime" to be that range of fields and states in which the quadratic term $(e \overrightarrow{\mathscr{A}} / c)^{2}$ is significant, but still relatively weak, i.e., its effects are observable, but they can be calculated or at least estimated using perturbation theory. This regime is the main subject of this paper.

As the magnetic field strength is increased further, our calculations have shown that classical orbits of the electron do not retain their orderly, multiply periodic behavior; they become irregular or chaotic. It is believed that this implies that the quantum-mechanical energy spectrum will also be irregular. ${ }^{4}$

For very strong magnetic fields, there is another regime in which orbits are regular, but totally different from familiar elliptical orbits. ${ }^{3}$ If the field is so strong that the magnetic force on the electron is much larger than the electrostatic force, then the electron will move on an approximately helical path. Hence the atom must have the shape of a long tube, with the electron spiraling rapidly around a field line and traveling slowly back and forth along the field line. We hope to study these trajectories and their associated quantum states in the future.

In this paper we present the results of our studies of the "quadratic region," where $(e \overrightarrow{\mathscr{A}} / c)^{2}$ is significant but not too large. The primary motivation of this paper is to interpret the spectrum of energy levels that was observed in Kleppner's laboratory at Massachusetts Institute of Technology (MIT). ${ }^{5}$

After specifying the coordinates and Hamiltonian in Sec. II, we will use perturbation theory to calculate classical orbits in Sec. III. The results will be compared with exact, numerically computed orbits in Sec. IV. Then in Sec. V we quantize the action variables associated with these orbits to obtain a discrete energy spectrum. Excellent agreement is found with observations and calculations made at MIT.

\section{DEFINITIONS, COORDINATES, AND HAMILTONIAN}

We take the nucleus to be infinitely massive, and at rest. Let $\vec{r}$ represent the position of the electron relative to the nucleus, with length $r$.

In a space-fixed frame with unit vectors $\hat{i}, \hat{j}, \hat{k}$, let $x^{\prime}, y^{\prime}, z^{\prime}$ be the components of $\vec{r}$, and let $\vec{p}$ be the canonical 
momentum of the electron, with components $p_{x^{\prime}}, p_{y^{\prime}}, p_{z^{\prime}}$.

For a uniform magnetic field in the $+z^{\prime}$ direction, $\overrightarrow{\mathbf{B}}=B \hat{k}$, the vector potential is

$$
\begin{aligned}
\overrightarrow{\mathscr{A}} & =-\frac{1}{2} \overrightarrow{\mathbf{r}} \times \overrightarrow{\mathbf{B}} \\
& =-\frac{1}{2} \boldsymbol{B}\left(y^{\prime} \hat{i}-x^{\prime} \hat{j}\right)
\end{aligned}
$$

and the Hamiltonian for the electron of $\operatorname{mass}^{6} \mu$ is

$$
\begin{aligned}
H^{\prime}= & \frac{1}{2 \mu}(\overrightarrow{\mathrm{p}}+e \overrightarrow{\mathscr{A}} / c)^{2}-Z e^{2} / r \\
= & p^{2} / 2 \mu+e B L_{z^{\prime}} / 2 \mu c+e^{2} B^{2}\left(x^{\prime 2}+y^{\prime 2}\right) / 8 \mu c^{2} \\
& -Z e^{2} / r
\end{aligned}
$$

The main effect of a magnetic field is to cause the plane of the orbit to precess at the Larmor frequency $\omega_{L}=e B / 2 \mu c$, and the Hamiltonian is simplified if we transform to a frame of reference that is rotating the same way. Let

$$
\begin{aligned}
& x=x^{\prime} \cos \omega_{L} t+y^{\prime} \sin \omega_{L} t, \\
& y=-x^{\prime} \sin \omega_{L} t+y^{\prime} \cos \omega_{L} t, \\
& z=z^{\prime} .
\end{aligned}
$$

In classical mechanics, the appropriate canonical transformation has the generating function

$$
\begin{aligned}
W_{L}= & p_{x}\left(x^{\prime} \cos \omega_{L} t+y^{\prime} \sin \omega_{L} t\right) \\
& +p_{y}\left(-x^{\prime} \sin \omega_{L} t+y^{\prime} \cos \omega_{L} t\right)+p_{z} z^{\prime}
\end{aligned}
$$

from which the transformed Hamiltonian is obtained by the usual rules

$$
\begin{aligned}
p_{i}^{\prime} & =\partial W_{L} / \partial x_{i}^{\prime}, \quad x_{i}=\partial W_{L} / \partial p_{i}, \\
H & =H^{\prime}+\partial W_{L} / \partial t .
\end{aligned}
$$

A straightforward calculation gives

$$
\begin{aligned}
H=p^{2} / 2 \mu-k / r+\lambda\left(x^{2}+y^{2}\right), & \\
k & =Z e^{2}, \lambda=e^{2} B^{2} / 8 \mu c^{2} .
\end{aligned}
$$

In this precessing frame, the term linear in $B$ has disappeared, and the Hamiltonian contains a cylindrically symmetric attractive effective potential-energy proportional to $B^{2}$.

In Sec. III we use standard methods of celestial mechanics to find the evolution of the orbit, assuming that the $B^{2}$ term is weak compared to the Coulomb term. ${ }^{7}$

\section{DESCRIPTION OF CLASSICAL MOTION BY PERTURBATION THEORY}

In perturbation theory, the trajectory of the electron may be described as a Kepler ellipse with orbital parameters that evolve slowly in time. For this purpose, Kepler action and angle variables provide the simplest canonical momenta and coordinates.

\section{A. Action and angle variables for unperturbed Kepler ellipses}

Action and angle variables for the Kepler problem ${ }^{8}$ are given in Table $I$ and indicated in Fig. 1, in the rotating $x, y, z$ coordinate system.

The plane of the orbit intersects the $x y$ plane in the line of nodes; the ascending node is the point at which the electron passes through the $x y$ plane from negative to positive

TABLE I. Variables describing Kepler ellipses.

\begin{tabular}{ll}
\hline \hline Action-angle variables & \\
$I_{1} \equiv L_{z}$ & $\begin{array}{l}z \text { component of orbital angular momentum } \\
I_{2} \equiv L\end{array}$ \\
$I_{3}=\left(\mu k^{2} /-2 H_{0}\right)^{1 / 2}$ & $\begin{array}{l}\text { Magnitude of orbital angular momentum; } I_{2}>\left|I_{1}\right| \\
\text { Principal action, related to the Kepler energy, } \\
\text { and corresponding to the principal quantum } \\
\text { number; } I_{3}>I_{2}\end{array}$ \\
$\phi_{1}$ & Longitude of ascending node \\
$\phi_{2}$ & Argument of perihelion \\
$\phi_{3}$ & Mean anomaly, related to the true anomaly $\chi$ \\
Other variables & \\
$i=\cos ^{-1}\left(L_{z} / L\right)$ & Polar angle specifying orientation of angular \\
$=\cos ^{-1}\left(I_{1} / I_{2}\right)$ & momentum vector \\
$\Phi=\phi_{1}-\pi / 2$ & Corresponding azimuthal angle \\
$\chi$ & True anomaly, or geometric angle between Laplace \\
$a=-k / 2 H_{0}=I_{3}^{2} / \mu k$ & vector and instantaneous position of particle \\
$b=\left(a L^{2} / \mu k\right)^{1 / 2}$ & Major semiaxis \\
$=I_{2} I_{3} / \mu k$ & Minor semiaxis \\
$e=\left(1-b^{2} / a^{2}\right)^{1 / 2}$ &
\end{tabular}




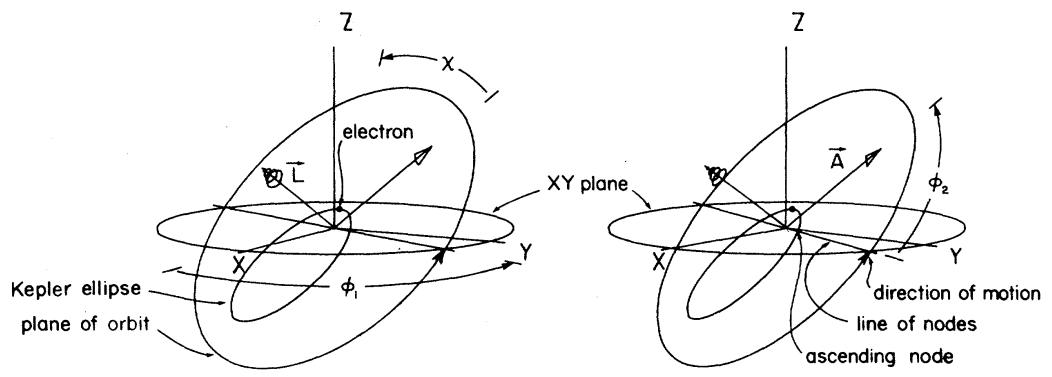

FIG. 1. Kepler orbit in space.

$z$, and $\phi_{1}$ is the angle between the $x$ axis and the ascending node. The angular momentum vector is distinguished by a canonical arrowhead, and it is perpendicular to the plane of the orbit. Its polar and azimuthal angles are $i$ and $\Phi$. The Laplace (or Runge-Lenz) vector

$$
\overrightarrow{\mathrm{A}}=\overrightarrow{\mathrm{p}} \times \overrightarrow{\mathrm{L}}-\mu k \overrightarrow{\mathrm{r}} / r
$$

points in the direction of the perihelion and has a magnitude which is proportional to the eccentricity $e$ of the orbit; it is shown with a flat arrowhead in Fig. 1. The argument of the perihelion $\phi_{2}$ is the angle, measured in the plane of the orbit, between the ascending node and the Laplace vector. The "true anomaly" $\chi$ is the angle between the Laplace vector and the instantaneous position of the particle (again measured in the plane of the orbit). The angle variable $\phi_{3}$ is called the "mean anomaly": Like $\chi$, it increases by $2 \pi$ every time the particle goes around one cycle, but unlike $\chi, \phi_{3}$ increases linearly with time. All three angle variables $\phi_{1}, \phi_{2}, \phi_{3}$ have the range $[0,2 \pi)$.

Action variables in the Kepler problem (Table I) are restricted such that

$$
\left|I_{1}\right| \leq I_{2} \leq I_{3} \text {. }
$$

The first of these is just $\left|L_{z}\right|<L$, and the second follows from the fact that the principal action is a sum of radial and angular actions. In quantum mechanics (8) corresponds to $|m| \leq l \leq n-1$.

Geometrical information is extracted from action-angle variables as follows. The polar angle $i$ between the angular momentum vector and the $z$ axis is given by $\cos ^{-1}\left(L_{z} / L\right)=\cos ^{-1}\left(I_{1} / I_{2}\right)$, and the corresponding azimuthal angle is $\phi_{1}-\pi / 2$. Since these two angles specify the direction of the angular momentum vector, they define the plane of the orbit. Then $\phi_{2}$ tells the orientation of the ellipse in that plane. The major semiaxis of the ellipse, $a$, is a function only of the energy $a=-k / 2 H_{0}$, so it is calculable from $I_{3}$, and the minor semiaxis is calculated from $a$ and the angular momentum.

Conversely, given the instantaneous position $\vec{r}$ and momentum $\overrightarrow{\mathrm{p}}$ of the particle, Kepler actions and angles can be calculated by the following procedure. From $\vec{r}$ and $\overrightarrow{\mathrm{p}}$, compute the vector angular momentum $\overrightarrow{\mathrm{L}}$ and the Kepler energy $H_{0}=p^{2} / 2 m-k / r$. This gives $I_{1}, I_{2}, I_{3}, \Phi$, $i$, and $\phi_{1}=\Phi+\pi / 2$. The Laplace vector can be computed from (7), and, using its components, we can calculate polar and azimuthal angles $\theta_{A}$ and $\phi_{A}$, giving the direction of $\overrightarrow{\mathrm{A}}$. By perusing Goldstein's figure $10.5,{ }^{8}$ we find

$$
\phi_{2}=\cos ^{-1}\left[\sin \theta_{A} \cos \left(\phi_{A}-\phi_{1}\right)\right]
$$

and

$$
\phi_{3}=\psi-e \sin \psi,
$$

where $\psi$ is the "eccentric anomaly," related to the true anomaly $\chi$ by

$$
\tan \frac{1}{2} \chi=[(1+e) /(1-e)]^{1 / 2} \tan \frac{1}{2} \psi .
$$

The relationship between the set $\{\vec{r}, \vec{p}\}$ and the set $\left\{I_{n}, \phi_{n}\right\}$ is a canonical transformation, so it holds independently of the Hamiltonian. For the Kepler Hamiltonian, $I_{1}, I_{2}, I_{3}, \phi_{1}$, and $\phi_{2}$ are constants of the motion, whereas for the full Hamiltonian these quantities could change with time, but the equations transforming $\overrightarrow{\mathrm{r}}$ and $\overrightarrow{\mathrm{p}}$ into $I^{\prime} \mathrm{s}$ and $\phi$ 's hold in any case.

\section{B. Effective Hamiltonian and conservation laws}

Equations of motion are obtained by reexpressing the Hamiltonian (6) in terms of action and angle variables, and evaluating the canonical equations

$$
\dot{\phi}_{j}=\partial H / \partial I_{j}, \quad \dot{I}_{j}=-\partial H / \partial \phi_{j} \text {. }
$$

In perturbation theory, the Hamiltonian (6) is divided into zeroth-order and perturbing terms

$$
H=H_{0}+\lambda H_{1},
$$

and the canonical equations are averaged over one cycle $\tau$ of the unperturbed Kepler motion

$$
\begin{aligned}
\frac{\Delta \phi_{j}}{\tau} & =\tau^{-1} \int_{0}^{\tau} \frac{\partial H}{\partial I_{j}} d t \\
& =\frac{\partial}{\partial I_{j}}\left[\frac{\lambda}{\tau} \int_{0}^{\tau} H_{1} d t\right], j=1,2 \\
\frac{\Delta I_{j}}{\tau} & =-\tau^{-1} \int_{0}^{\tau} \frac{\partial H}{\partial \phi_{j}} d t \\
& =-\frac{\partial}{\partial \phi_{j}}\left[\frac{\lambda}{\tau} \int_{0}^{\tau} H_{1} d t\right], j=1,2,3 .
\end{aligned}
$$

Equations (11b) and (11d) hold because $H_{0}$ and $\tau$ depend only on $I_{3}$, not on the other actions or angles. [Equation (11b) does not hold for $j=3$; the equation for $\Delta \phi_{3} / \tau$ has other terms, but we do not use that equation.]

The average development in time of $I_{1}, I_{2}, I_{3}, \phi_{1}$, and $\phi_{2}$ is therefore given by canonical equations having the effective Hamiltonian 


$$
\lambda \bar{H}_{1}\left(I_{2}, \phi_{2} ; I_{1}, I_{3}\right)=\frac{\lambda}{\tau} \int_{0}^{\tau} H_{1} d t .
$$

This effective Hamiltonian is evaluated in Appendix A, and the result is

$$
\begin{aligned}
\lambda \bar{H}_{1}= & \left(\lambda I_{3}^{2} / 4 \mu^{2} k^{2} I_{2}^{2}\right) \\
& \times\left[\left(I_{1}^{2}+I_{2}^{2}\right)\left(5 I_{3}^{2}-3 I_{2}^{2}\right)\right. \\
& \left.+5\left(I_{3}^{2}-I_{2}^{2}\right)\left(I_{2}^{2}-I_{1}^{2}\right) \cos 2 \phi_{2}\right] .
\end{aligned}
$$

Equations of motion for $I$ 's and $\phi$ 's are given in Appen$\operatorname{dix} B$, but we can get most of the information we need from conservation laws. The effective Hamiltonian $\lambda \bar{H}_{1}$ is independent of $\phi_{1}$ because the magnetic term $\lambda\left(x^{2}+y^{2}\right)$ is cylindrically symmetric; hence $I_{1} \equiv L_{z}$ is conserved. This holds not only in first order, but also for the exact motion. $\bar{H}_{1}$ is also necessarily independent of $\phi_{3}$, because we have averaged over one closed cycle of the unperturbed motion, and therefore $I_{3}$ is, to first order, a constant of the motion. Since $I_{3}$ is related to $H_{0}$, and $H_{0}$ is related to the major axis and the period of the Kepler ellipse, it follows that the ellipse evolves under the perturbation in such a way that its major axis, its zeroth-order energy, and the period of motion of the particle around the ellipse, do not change with time.

A third constant of the motion relates the time development of $I_{2}$ to that of $\phi_{2}$. This third conserved quantity is of course the effective Hamiltonian $\lambda \bar{H}_{1}$ itself. In their measurements of the spectrum of high Rydberg states of an atom in a magnetic field, Kleppner and his collaborators ${ }^{5}$ found evidence that there may be three conserved quantities associated with the motion. In the present formulation these conserved quantities are found to be $I_{1}$, $\bar{H}_{1}$, and $I_{3}$. An alternative set of conserved quantities is $H, L_{z}$, and $\bar{H}_{0}$; the first two of these are exactly conserved, but $\bar{H}_{0}$ is conserved only to first order in perturbation theory. We already mentioned that when the strength of the magnetic field is increased, the trajectories become irregular; this implies that a third integral of the motion does not in general exist, so $H_{0}$ cannot in general be conserved.

\section{Evolution of orbital parameters}

As stated above, in the present framework we describe the trajectory as an ellipse whose parameters evolve slowly with time. Mathematically, the simplest representation is in terms of action-angle variables, but this description requires some translation in order to become physically meaningful. We present the mathematical description here.

We showed that $I_{1}$ and $I_{3}$ are conserved, but $I_{2}$ and $\phi_{2}$ change with time. Their time derivatives are independent of $\phi_{1}$, so $\phi_{1}$ is an ignorable coordinate, and we can discuss the motion within the $\left(I_{2}, \phi_{2}\right)$ phase plane. We have therefore reduced the problem to two canonical equations, or one degree of freedom. Furthermore, we know that the motion in the $\left(I_{2}, \phi_{2}\right)$ plane is such that $\bar{H}_{1}$ is conserved. Hence if we draw a contour plot of $\bar{H}_{1}$ as a function of $I_{2}$ and $\phi_{2}$ for fixed $I_{1}$ and $I_{3}$, then $I_{2}$ and $\phi_{2}$ develop together in time such that the system follows a contour of constant $\bar{H}_{1}$.

Contours can be calculated by solving the equation

$$
\bar{H}_{1}\left(I_{2}, \phi_{2} ; I_{1}, I_{3}\right)=\bar{H}_{1}^{0}
$$

for $\phi_{2}$ as a function of $I_{2}$,

$$
\cos 2 \phi_{2}=\frac{\left(2 I_{2}^{2} \bar{H}_{1}^{0} / D\right)-\left(I_{1}^{2}+I_{2}^{2}\right)\left(5 I_{3}^{2}-3 I_{2}^{2}\right)}{5\left(I_{2}^{2}-I_{1}^{2}\right)\left(I_{3}^{2}-I_{2}^{2}\right)},
$$

where

$$
D=I_{3}^{2} / 2 \mu^{2} k^{2} .
$$

Alternatively, to obtain $I_{2}$ as a function of $\phi_{2}$, one solves the quadratic equation

$$
\begin{aligned}
& \quad a I_{2}^{4}+b I_{2}^{2}+c=0, \\
& \text { where } \\
& \qquad \begin{aligned}
a= & 5 \cos 2 \phi_{2}+3, \\
b= & \left(2 \bar{H}_{1}^{0} / D\right)-5 I_{3}^{2}\left(1+\cos 2 \phi_{2}\right) \\
& \quad-I_{1}^{2}\left(5 \cos 2 \phi_{2}-3\right), \\
c= & 5 I_{1}^{2} I_{3}^{2}\left(\cos 2 \phi_{2}-1\right) .
\end{aligned}
\end{aligned}
$$

Such contour plots are shown in Fig. 2. In all of these plots $I_{3}=30 \hbar$, while $\left|I_{1}\right|=0,1,5,10,15$, and $25 \hbar$. From Eq. (13), we see that $\bar{H}_{1}$ is periodic in $\phi_{2}$ with period $\pi$, and it depends only on $I_{1}^{2}$; hence these plots repeat themselves in every interval $[n \pi,(n+1) \pi]$, and plots for $\pm I_{1}$ are identical. Also, the plots are symmetric about $\pi / 2$.

$$
\text { 1. } I_{1}=1
$$

Let us examine the graph with $I_{1}=1$ first (in the remainder of Sec. III C, the factor of $\hbar$ for $I_{1}, I_{2}$, and $I_{3}$ will be omitted). In this case $I_{2}$ must fall between 1 and 30 , so there is a forbidden region between 0 and 1 . When $I_{2}=I_{1}, \bar{H}_{1}$ is independent of $\phi_{2}$ and is given by ${ }^{9}$

$$
\bar{H}_{1}^{\max }=\left(I_{3}^{2} / 2 \mu^{2} k^{2}\right)\left(5 I_{3}^{2}-3 I_{1}^{2}\right)
$$

which turns out to be the maximum value of $\bar{H}_{1}, \bar{H}_{1}$ is also independent of $\phi_{2}$ on the line $I_{2}=I_{3}$, where it has the value

$$
\bar{H}_{1}^{\text {crit }}=\left(I_{3}^{2} / 2 \mu^{2} k^{2}\right)\left(I_{1}^{2}+I_{3}^{2}\right) .
$$

$\bar{H}_{1}$ has a minimum when $\partial \bar{H}_{1} / \partial \phi_{2}=0$ and $\partial \bar{H}_{1} / \partial I_{2}=0$,

$$
\begin{aligned}
& \phi_{2}=\phi_{2}^{m}=\pi / 2, \\
& I_{2}=I_{2}^{m}=\left(I_{1} I_{3} \sqrt{5}\right)^{1 / 2}, \\
& \bar{H}_{1}^{\min }=\left(I_{3}^{2} / 2 \mu^{2} k^{2}\right)\left(2 I_{1}\right)\left(\sqrt{5} I_{3}-2 I_{1}\right) .
\end{aligned}
$$

This is a point of stable equilibrium; in Fig. $2(\mathrm{~b})$ it is at $\phi_{2}=\pi / 2, I_{2}=8.19$.

Around the equilibrium point, contours of constant $\bar{H}_{1}$ form closed loops. As time increases, a phase point would move clockwise around a loop, moving most rapidly where contours are close together, near the bottom of the loops. We refer to motion around these loops as "libration."

Other contours in Fig. 2(b) are open, extending from one side of the figure to the other. A phase point moves on these curves in a generally leftward sense, from $\pi$ to 0 . We refer to this motion as "rotation."

Between rotation and libration curves is a $U$-shaped separatrix. It joins the line $I_{2}=I_{3}$ at two " $T$ points," so the value of $\bar{H}_{1}$ on the separatrix is $\bar{H}_{1}^{\text {crit }}$, and, from (15), the separatrix satisfies the equation

$$
\cos 2 \phi_{2}=\left(I_{1}^{2}+\frac{3}{5} I_{2}^{2}\right) /\left(I_{1}^{2}-I_{2}^{2}\right) \text {. }
$$



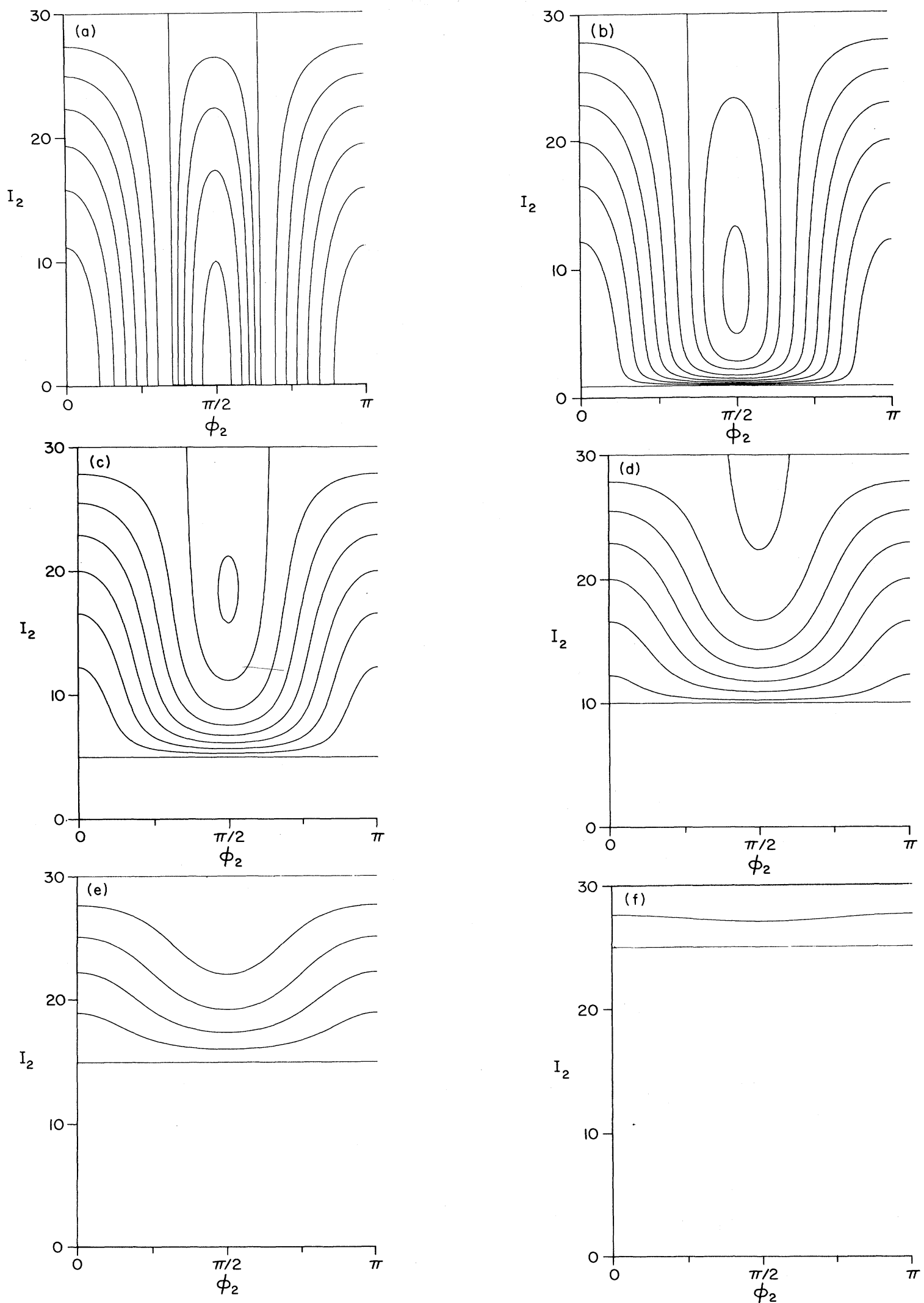

FIG. 2. (a) Contours of constant $\bar{H}_{1}\left(I_{2}, \phi_{2} ; I_{1}, I_{3}\right)$. In all cases $I_{3} / \hbar=n=30$, and $I_{2}$ corresponds to $L$. Here $I_{1}=0$. Abscissa in units of $\hbar$. (b) $I_{1}=1 \hbar$. (c) $I_{1}=5 \hbar$. (d) $I_{1}=10 \hbar$. (e) $I_{1}=15 \hbar$. (f) $I_{1}=25 \hbar$. 
The $T$ points occur at

$$
\phi_{2}=\phi_{2}^{T}=\frac{1}{2} \cos ^{-1}\left[\left(I_{1}^{2}+\frac{3}{5} I_{3}^{2}\right) /\left(I_{1}^{2}-I_{3}^{2}\right)\right],
$$

where $\partial \bar{H}_{1} / \partial I_{2}=0$, and the lowest point of the separatrix is at $\phi_{2}=\pi / 2$ and

$$
I_{2}=I_{2}^{U}=\sqrt{5} I_{1} \text {. }
$$

Each of the $T$ points is an unstable equilbrium, and since they occur when $I_{2}=I_{3}$, this means that circular orbits are unstable if $I_{1}$ is small. The motion of a phase point around the separatrix is also clockwise, starting at the right-hand $T$ point at $t \rightarrow-\infty$ and approaching the lefthand $T$ point as $t \rightarrow+\infty$. Points close to the separatrix but inside it move along the loops, while those outside it follow the rotational motion.

$$
\text { 2. } 1 \leq I_{1} \leq I_{3}
$$

If $I_{1}$ increases, the allowed region (8) decreases in size, and the equilibrium point, the loops, and the separatrix move upward in accordance with Eqs. (20b), (21), and (23). Also the range of values of $\bar{H}_{1}$ between $\bar{H}_{1}^{\min }$ and $\bar{H}_{1}^{\max }$ gets smaller. This is indicated in the plots with $I_{1}=5$ and 10. As $I_{1}$ is increased further, the equilibrium point, the loops, and the separatrix all cease to exist. Equations (20b) and (23) tell us that the equilibrium point and the bottom of the separatrix simultaneously pass through the upper boundary of the diagram when

$$
I_{1}=\hat{I}_{1}=I_{3} / \sqrt{5} \text {. }
$$

For larger $I_{1}$, only rotational motion occurs. For $I_{1}$ close to $I_{3}, I_{2}$ remains nearly constant on the $\bar{H}_{1}$ contours and phase points move with $\phi_{2}$ decreasing at a nearly constant rate. For these larger values of $I_{1}$, circular orbits are no longer unstable.

$$
\text { 3. } I_{1}=0
$$

The case $I_{1}=0$ is trickier, because in these variables, the motion is actually discontinuous. For $I_{1}=0$, the equilibrium point (20b) is at $I_{2}=0$, and the separatrices are at

$$
\phi_{2}=\phi_{2}^{S_{0}}=\frac{1}{2} \cos ^{-1}\left(-\frac{3}{5}\right)=1.11 \text { and } 2.03 \text {. }
$$

Only upper parts of loops appear in Fig. 2(a), and the motion around a semiloop is clockwise, beginning to the left of $\pi / 2$ and ending to the right.

The rotational curves have been broken into two parts by the separatrices, and a phase point that starts at large $I_{2}$ and $\phi_{2}=\pi$ moves toward decreasing $\phi_{2}$ and $I_{2}$, while a point starting at small $\phi_{2}$ and $I_{2}=0$ moves toward increasing $I_{2}$ and decreasing $\phi_{2}$.

What happens at $I_{2}=0$ ? Classical mechanics does not allow piling up of phase points (density in phase space is conserved). Hence a phase point approaching $I_{2}=0$ and, say, $\phi_{2}=2.36$ must disappear and reappear somewhere else on a curve having the same value of $\bar{H}_{1}$. We can understand this only by thinking of the case $I_{1}=0$ as the limit of $I_{1}$ small but nonzero. Then we can see that this discontinuous motion is the limit of the very quick passage of the phase point through $\pi / 2$ that is apparent on the plot for $I_{1}=1$ [Fig. 2(b)]. This leads us to the rule that when the phase point touches $I_{2}=0$, it is reflected through $\pi / 2$,

$$
\phi_{2} \rightarrow \pi-\phi_{2} \text {. }
$$

The same thing happens near $3 \pi / 2$.

\section{Evolution of $\phi_{1}$}

Let us now examine the time development of the previously ignored coordinate $\phi_{1}$, the longitude of the ascending node. Its evolution is governed by the equation of motion (B1). It is easy to show that when we average over a Kepler cycle (indicated by angular brackets), then $\left\langle d \phi_{1} / d t\right\rangle \geq 0$, so $\phi_{1}$ increases in every Kepler cycle. Furthermore $\partial\left\langle d \phi_{1} / d t\right\rangle / \partial I_{2} \leq 0$ so the rate of increase of $\phi_{1}$ is larger when $I_{2}$ is smaller. When $I_{1}$ is small, $\left\langle d \phi_{1} / d t\right\rangle$ is small unless $I_{2}$ is also small.

We have found an interesting approximate degeneracy between the periods of motion of $\phi_{1}$ and $\phi_{2}$. Let $\tau_{1}$ be the period for $\phi_{1}$, so $\phi_{1}$ increases by $2 \pi$ in the time $\tau_{1}$. Then for the rotational orbits, $\phi_{2}$ decreases by $2 \pi$ in about the same period,

$$
\tau_{2}^{\text {rot }} \simeq \tau_{1} .
$$

For librational orbits, the time required for $\phi_{2}$ and $I_{2}$ to move around a loop in Fig. 2(b) is

$$
\tau_{2}^{\mathrm{vib}} \simeq \frac{1}{2} \tau_{1}
$$

This approximate degeneracy holds when $I_{1}$ is sufficiently small, but not in general. In fact, in the opposite limit $I_{1} \rightarrow I_{3}$, one finds from Eqs. (B1) and (B2) a different degeneracy,

$$
\left\langle\frac{d \phi_{1}}{d t}\right\rangle=\frac{\lambda I_{3}^{3}}{\mu^{2} k^{2}},\left\langle\frac{d \phi_{2}}{d t}\right\rangle=-\frac{4 \lambda I_{3}^{3}}{\mu^{2} k^{2}},
$$

so $\phi_{2}$ decreases by $2 \pi$ when $\phi_{1}$ increases by $\pi / 2$,

$$
\tau_{2}=\frac{1}{4} \tau_{1} \text {. }
$$

\section{EXACT CLASSICAL MOTION}

Above, first-order perturbation theory was used to calculate and describe trajectories of the Hamiltonian (6). We have also computed essentially exact classical trajectories, using numerical integration of Hamilton's equations. The computations presented in this section verify that first-order perturbation theory is accurate for states around $n=30$ and fields less than $6 \mathrm{~T}$. In addition, these exact trajectories provide an alternative basis for semiclassical calculation of the energy spectrum, which we will present later.

In cylindrical coordinates,

$$
\rho=\left(x^{2}+y^{2}\right)^{1 / 2}, \phi=\tan ^{-1}(y / x), \quad z=z,
$$

the Hamiltonian (16) is

$$
\begin{aligned}
H= & (2 \mu)^{-1}\left(p_{\rho}^{2}+p_{z}^{2}+p_{\phi}^{2} / \rho^{2}\right) \\
& -k /\left(\rho^{2}+z^{2}\right)^{1 / 2}+\lambda \rho^{2} .
\end{aligned}
$$

$p_{\phi} \equiv L_{z}$ is a constant of the motion, $\phi$ is an ignorable coordinate, and the equations of motion are

$$
\begin{aligned}
& \dot{\rho}=p_{\rho} / \mu, \quad \dot{z}=p_{z} / \mu, \\
& \dot{p}_{\rho}=-k \rho /\left(\rho^{2}+z^{2}\right)^{3 / 2}+p_{\phi}^{2} / \mu \rho^{3}-2 \lambda \rho, \\
& \dot{p}_{z}=-k z /\left(\rho^{2}+z^{2}\right)^{3 / 2}, \\
& \dot{\phi}=p_{\phi} / \mu \rho^{2}, \quad \dot{p}_{\phi}=0 .
\end{aligned}
$$



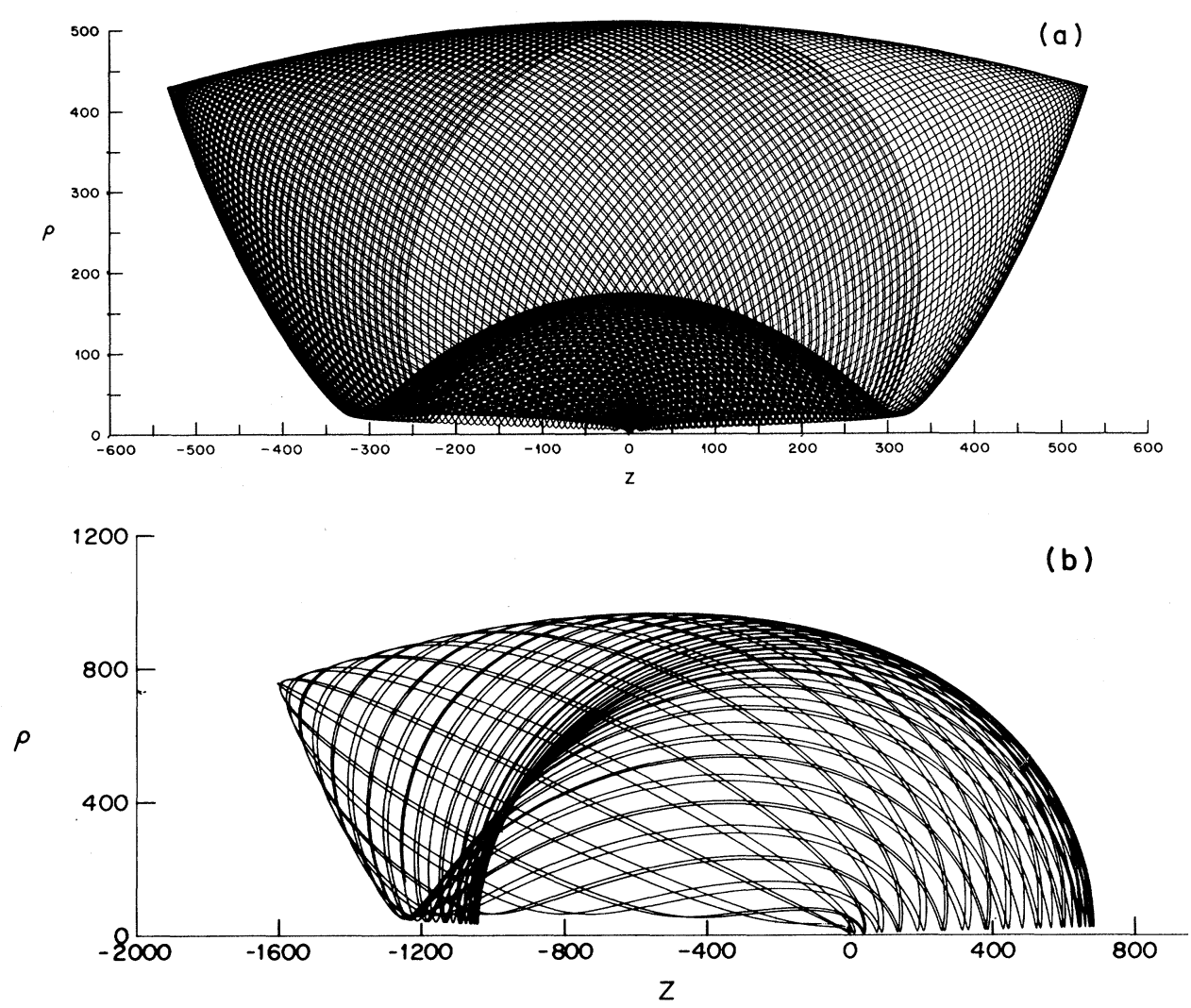

FIG. 3. (a) Rotating trajectory in $\rho, z$ coordinates (a.u.). (b) Librating trajectory in $\rho, z$ coordinates (a.u.).

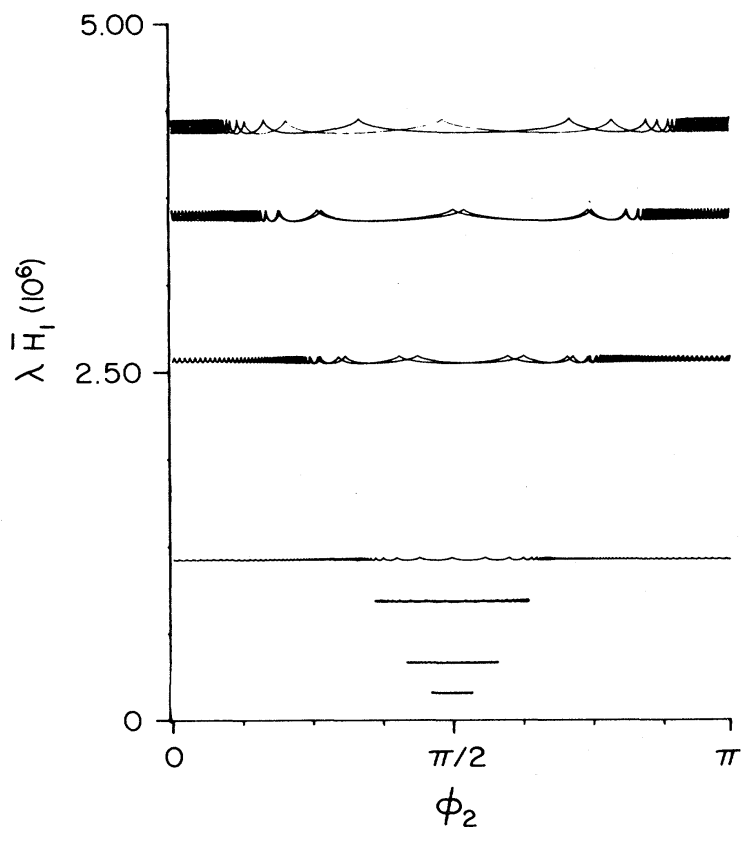

FIG.4. $\bar{H}_{1}(t)$ vs $\phi_{2}(t)$ for exact numerically computed trajectories $(B=1 \mathrm{~T})$.
Trajectories were drawn on a two-dimensional plot, with $\rho$ and $z$ as axes. When $L_{z}$ and $\lambda$ are both small, two types of trajectories are found, as indicated in Figs. 3(a) and $3(\mathrm{~b})$. The first of these corresponds to what we previously called a rotating trajectory, and the second is a librating trajectory. This correspondence was established by calculating instantaneous values of Kepler actions and angles from the instantaneous position and momentum of the particle, using the procedure given in the next-to-last paragraph of Sec. III A.

When projected into $(\rho, z)$ coordinates, a Kepler ellipse is folded. In Fig. 3(a), if one traces the orbit starting at $z=0$ and $\rho \sim 500$, near the center of the upper caustic, one sees the projection of a wide precessing ellipse. When the trajectory touches either of the upper corners, the ellipse is very narrow. Close examination reveals that on every Kepler cycle, the ellipse touches four caustics.

To verify that our first-order calculations are accurate, it is sufficient to show that $\bar{H}_{1}$ is approximately conserved on the exact trajectory. Again using the relationship between $\{\vec{r}, \vec{p}\}$ and $\left\{I_{n}, \phi_{n}\right\}$, we have calculated the value of $\bar{H}_{1}(t)$ as a function of $\phi_{2}(t)$ for several exact trajectories. The results plotted in Fig. 4 demonstrate that, as expected, $\bar{H}_{1}$ is not exactly conserved, but it has no secular change; it fluctuates slightly in each Kepler cycle, but after a long time it is still fluctuating in the same way about the same value.

Similarly, Fig. 5 shows $I_{2}(t)$ (angular momentum) vs 


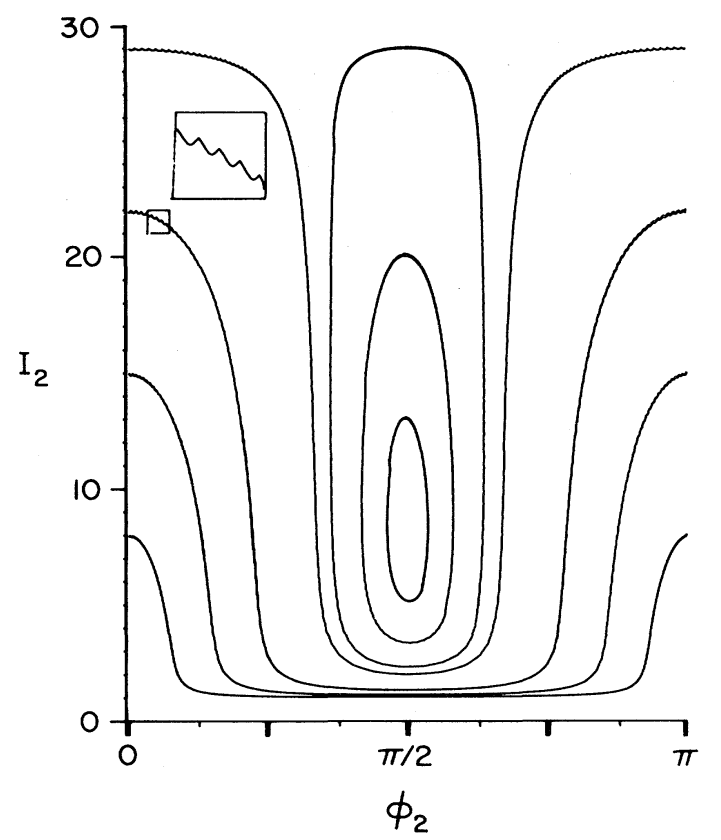

FIG. 5. $I_{2}(t)$ vs $\phi_{2}(t)$ for exact numerically computed trajectories $(B=1 \mathrm{~T})$.

$\phi_{2}(t)$ for some exact trajectories with $L_{z}=1$. If the plot is expanded, small fluctuations become visible, but the correspondence between this figure and Fig. 2(b) is obvious.

\section{SPECTRUM OF ENERGY LEVELS}

In Secs. III and IV, we displayed properties of classical trajectories of the Hamiltonian (6). With this information in hand, it is now quite simple to compute a spectrum of semiclassical energy levels of our Hamiltonian. Classical action variables can be calculated either from perturbation theory or from the exact numerically calculated trajectories, and in either case, the spectrum is obtained by quantizing the action variables.

\section{A. Action variables and quantization conditions for exact trajectories}

It turns out that we cannot give a correct quantization prescription in perturbation theory until we examine the trajectory in $\rho, z$ coordinates. Hence it is most convenient to consider exact trajectories first.

As is well known, ${ }^{10}$ action variables are defined as

$$
A_{i}=\oint_{C_{i}} \sum_{k} p_{k} d q_{k}
$$

on topologically distinct paths $C_{i}$. An outline of a rotating trajectory is shown in Fig. 6(a); $C_{z}$ is a path on which $\rho$ is constant, and $p_{z}$ as a function of $z$ can be obtained numerically as a Poincaré surface of section (every time the numerically computed trajectory passes through $\rho=\rho_{0}$ in the positive sense, the values of $p_{z}$ and $z$ are recorded by the computer). This gives the set of points shown in Fig. $7(a)$, and the action variable ${ }^{11}$

$$
A_{z}=\oint_{C_{z}} p_{z} d z
$$
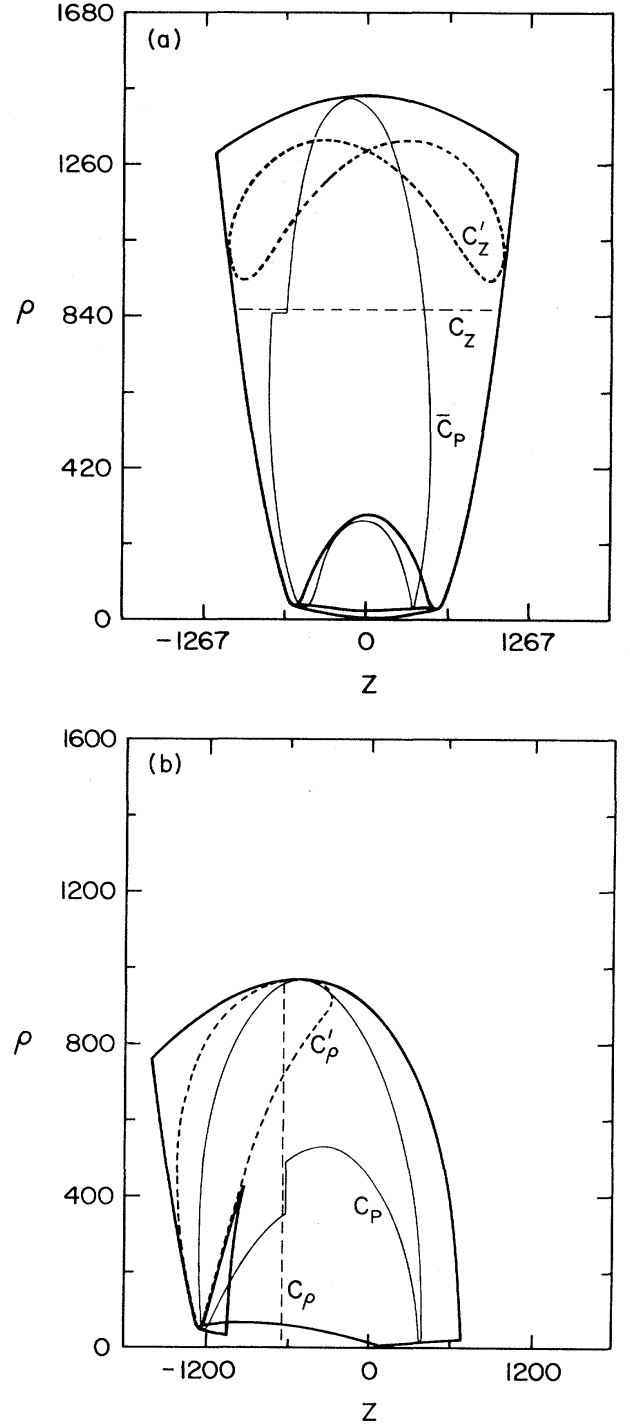

FIG. 6. (a) Outline of a rotating trajectory, and integration paths $C_{z}, C_{z}^{\prime}$, and $C_{p}$. (b) Outline of a vibrating trajectory, and integration paths $C_{\rho}, C_{\rho}^{\prime}$, and $\bar{C}_{P}$.

is the area inside the curve that is interpolated from the set of points. Now the path $C_{z}$ touches two caustics, and since they look like ordinary "fold" caustics ${ }^{12}$ we expect that each contributes $\pi / 2$ to the phase of the wave function. Hence the quantization condition is

$$
\oint_{C_{z}} p_{z} d z=\left(n_{z}+\frac{1}{2}\right) h,
$$

where $n_{z}$ is a (non-negative) integer.

The path $C_{p}$ in Fig. 6(a) is topologically distinct from $C_{z} . C_{p}$ is obtained by following the trjaectory itself from one Poincare surface point to the next, then closing the loop on the $\rho=\rho_{0}$ surface of section. This path touches four caustics, and since again each looks (more or less) like a fold caustic, we suppose that each contributes $\pi / 2$, and the quantization condition is 

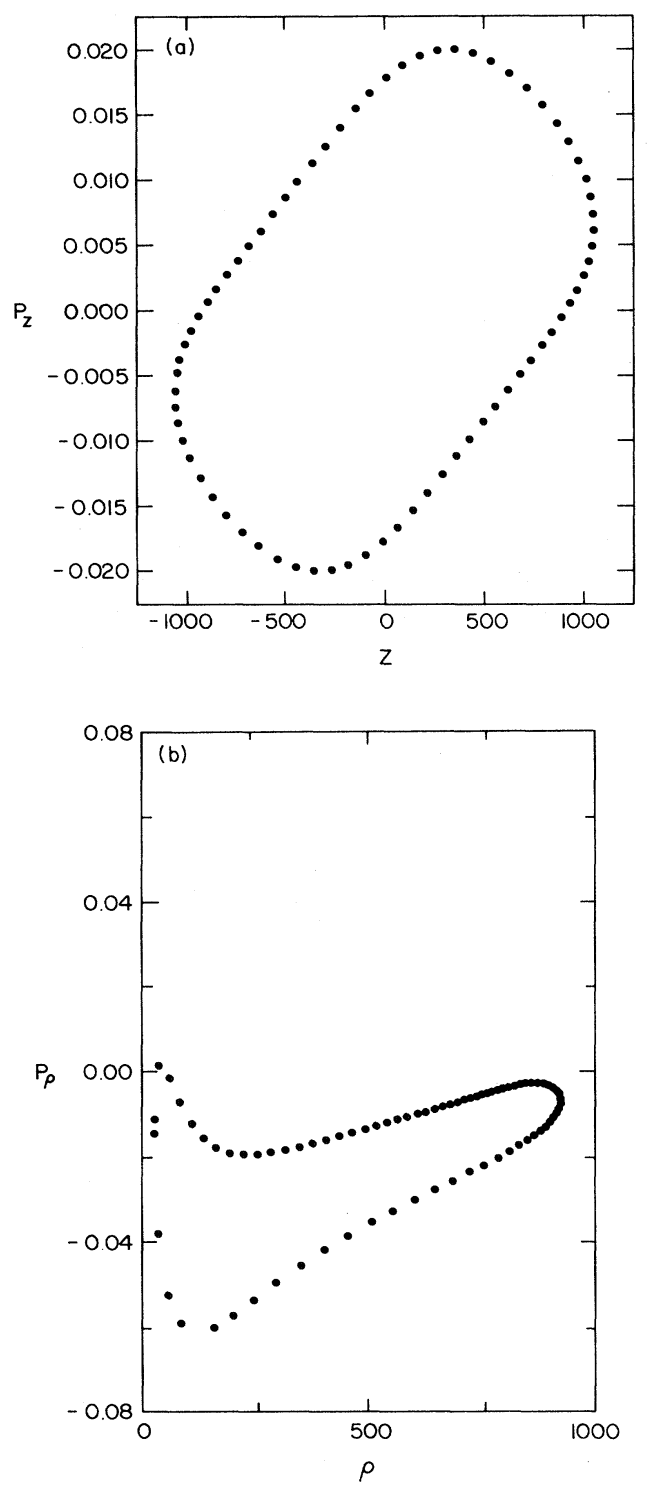

FIG. 7. (a) Poincaré surface of section for a rotating trajectory. Surface is $\rho=\rho_{0}$, and the graph shows $p_{z}$ as a function of $z$ on this surface. (b) Poincare surface of section for a librating trajectory. Surface is $z=z_{0}$, and the graph shows $p_{\rho}$ as a function of $\rho$.

$$
A_{p}=\oint_{C_{p}}\left(p_{\rho} d \rho+p_{z} d z\right)=n_{p} h,
$$

where $n_{p}$ is a positive integer (not zero).

Clearly $n_{p}$ is related to the principal quantum number $n$. The latter is proportional to the sum of radial and angular actions around an unperturbed Kepler ellipse

$$
\begin{aligned}
n \hbar & =I_{3}=\frac{1}{2 \pi} \oint p_{r} d r+p_{\theta} d \theta+p_{\phi} d \phi \\
& =\frac{1}{2 \pi} \oint p_{\rho} d \rho+p_{z} d z+p_{\phi} d \phi=n_{p} \hbar+L_{z},
\end{aligned}
$$

so

$$
n_{p}=n-m
$$

again confirming that $n_{p}$ should be a positive integer.

For a librating trajectory, shown in outline in Fig. 6(b), $C_{\rho}$ is a path on which $z$ is constant, and $\bar{C}_{p}$ is a path like $C_{p}$. By the same arguments, the quantum conditions are

$$
\begin{aligned}
& \oint_{C_{\rho}} p_{\rho} d \rho=\left(n_{\rho}+\frac{1}{2}\right) h, \\
& \oint_{\bar{C}_{p}} p_{\rho} d \rho+p_{z} d z=n_{p} h,
\end{aligned}
$$

where $n_{\rho}$ and $n_{p}$ are, respectively, non-negative and positive integers.

\section{B. Action variables from perturbation theory}

Quantization of classical perturbation theory requires additional thought, but it gives more information about the spectrum.

We showed in Sec. III B that $I_{1}$ and $I_{3}$ are, respectively, exact and approximate constants of the motion. It follows that they are quantized just as in the zeroth-order, unperturbed Kepler problem

$$
\begin{gathered}
I_{1}=m \hbar, \\
I_{3}=n \hbar
\end{gathered}
$$

with $m, n$ integers $|m| \leq n-1$. The third conserved quantity in the unperturbed problem is $I_{2}$, which would be quantized as $\left(l+\frac{1}{2}\right) \hbar$, but this is not conserved for the full Hamiltonian (6). In its place is the new action variable

$$
A_{2}=\oint I_{2} d \phi_{2}
$$

which is computed holding $I_{1}$ and $I_{3}$ fixed. For librating trajectories, this action variable is the area inside one of the loops in Fig. 2, while for rotating trajectories it is the area under one of the corresponding curves, extended from 0 to $2 \pi$. An analytic formula can be found for $A_{2}$ in terms of complete elliptic integrals, but it is more practical to evaluate the integral numerically.

\section{General properties of the spectrum}

Even before we compute these areas, we can see many qualitative properties of the spectrum. The total number of states for given $I_{1}$ and $I_{3}$ is $\hbar^{-1}$ times the area between the lines $I_{2}=I_{1}$ and $I_{2}=I_{3}$; i.e., it is $\left(I_{3}-I_{2}\right) / \hbar$ or $n-m$, of course. For every state, the correction $\Delta E$ to the unperturbed energy is positive, since $\lambda H_{1} \geq 0$. In perturbation theory, $\Delta E$ is linearly proportional to $\lambda$, i.e., the energy shift is proportional to the square of the magnetic field. The shift is greater than (20c) and less than (18),

$$
\lambda \bar{H}_{1}^{\min }<\Delta E<\lambda \bar{H}_{1}^{\max }
$$

so we have an upper and a lower bound on the spectrum.

Since there are two types of trajectory, there are two types of quantum states, which we again call librational and rotational. Librational levels are doubly degenerate: One group is associated with loops centered at $\pi / 2$ and the other with loops at $3 \pi / 2$. For the former, the wave function, like the trajectory, is mainly confined to a region close to the $-z$ axis; the latter group is oriented oppositely. Wave functions which are also eigenfunctions of the 
parity operator can be constructed as linear combinations of two degenerate librational wave functions, and presumably the degeneracy is split in higher order because of tunneling. (Numerical calculations presented later show that this splitting is very small.)

The total number of librational levels is proportional to the area inside the $U$-shaped separatrix. A quick calculation (Appendix C) tells us that this number is an even integer close to

$$
N_{L}=\frac{2}{\pi \hbar}\left[I_{3}\left(\phi_{2}^{T}-\frac{\pi}{2}\right]-I_{1} \cos ^{-1}\left(\frac{2 I_{1}}{\left(I_{3}^{2}-I_{1}^{2}\right)^{1 / 2}}\right]\right] .
$$

A somewhat simpler formula is obtained by approximating the $U$ by a rectangle; using (22) and (23) we immediately obtain

$$
N_{L} \simeq\left(I_{3}-\sqrt{5} I_{1}\right)\left(1-2 \phi_{2}^{T} / \pi\right) / \hbar .
$$

Rotational states are nondegenerate. They have higher energies than librational states, and adjacent states presumably have opposite parity. In the observed spectrum it should not be difficult to identify the transition between rotating and librating states. A phase point takes a very long time to travel along the separatrix, and rotating or librating paths near the separatrix have very long periods, or very low frequencies. From the correspondence between energy gaps and frequencies, we conclude that the two energy levels of given $n$ and $m$ that lie closest together correspond to trajectories that lie closest to the separatrix. Furthermore, the energy of the separatrix is given by (19), and this should also mark the boundary between librational and rotational levels.

\section{Quantization prescription in perturbation theory}

For quantitative calculation of the energy spectrum, a quantization condition is needed. We found that an accurate energy spectrum is obtained from the prescription

$$
A_{2}=\oint I_{2} d \phi_{2}=\left(n_{2}+\frac{1}{2}\right) h,
$$

i.e., the new action variable is quantized in half-integers for all states. One can "prove" that this prescription is correct by the following method. Consider first the rotating states. The new action variable can be written as

$$
A_{2}=\int_{0}^{2 \pi} I_{2} d \phi_{2}=\int I_{1} d \phi_{1}+I_{2} d \phi_{2}+I_{3} d \phi_{3},
$$

where the last integral is evaluated on a path on which $\phi_{1}$ and $\phi_{3}$ are constant and $\phi_{2}$ goes from 0 to $2 \pi$. From the canonical invariance of the form $\sum_{k} p_{k} d q_{k}$,

$$
A_{2}=\int p_{\rho} d \rho+p_{z} d z+p_{\phi} d \phi
$$

on a corresponding path in $\rho z \phi$ space. That corresponding path is obtained using equations given in Sec. III A, that relate $\left\{I_{n}, \phi_{n}\right\}$ to $(\overrightarrow{\mathrm{p}}, \overrightarrow{\mathrm{r}})$. For $\phi_{1}=0, \phi_{3}=\pi / 2$, and $\phi_{2}$ varying from 0 to $2 \pi$, one finds by numerical calculation that the projection of that path in $\rho, z$ coordinates is the closed loop labeled $C_{z}^{\prime}$ in Fig. 6(a). Moreover, from these calculations, or by careful thought and attention to Fig. 1, one finds that if $\phi_{2}$ increases by $2 \pi$ holding $\phi_{1}$ and $\phi_{3}$ fixed, then $\phi$ must increase by $2 \pi$. Therefore,

$$
A_{2}=\oint_{C_{z^{\prime}}} p_{\rho} d \rho+p_{z} d z+2 \pi L_{z} .
$$

Finally, the path $C_{z}^{\prime}$ is topologically equivalent to $C_{z}$, so

$$
\begin{aligned}
A_{2} & =\oint_{C_{z}} p_{z} d z+2 \pi L_{z}=A_{z}+2 \pi L_{z} \\
& =\left(n_{z}+\frac{1}{2}+m\right) h,
\end{aligned}
$$

i.e., for rotating states, $A_{2}$ is quantized in half-integers greater than $m$,

$$
n_{2}=n_{z}+m \text {. }
$$

For librating states, a similar analysis leads to the path $C_{\rho}^{\prime}$ in Fig. 6(b), and most of the same argument follows. Motion of $\phi_{2}$ around a librational loop leaves $\phi$ unchanged, so for librating states

$$
A_{2}=\left(n_{\rho}+\frac{1}{2}\right) h
$$

TABLE II. Quantization conditions.

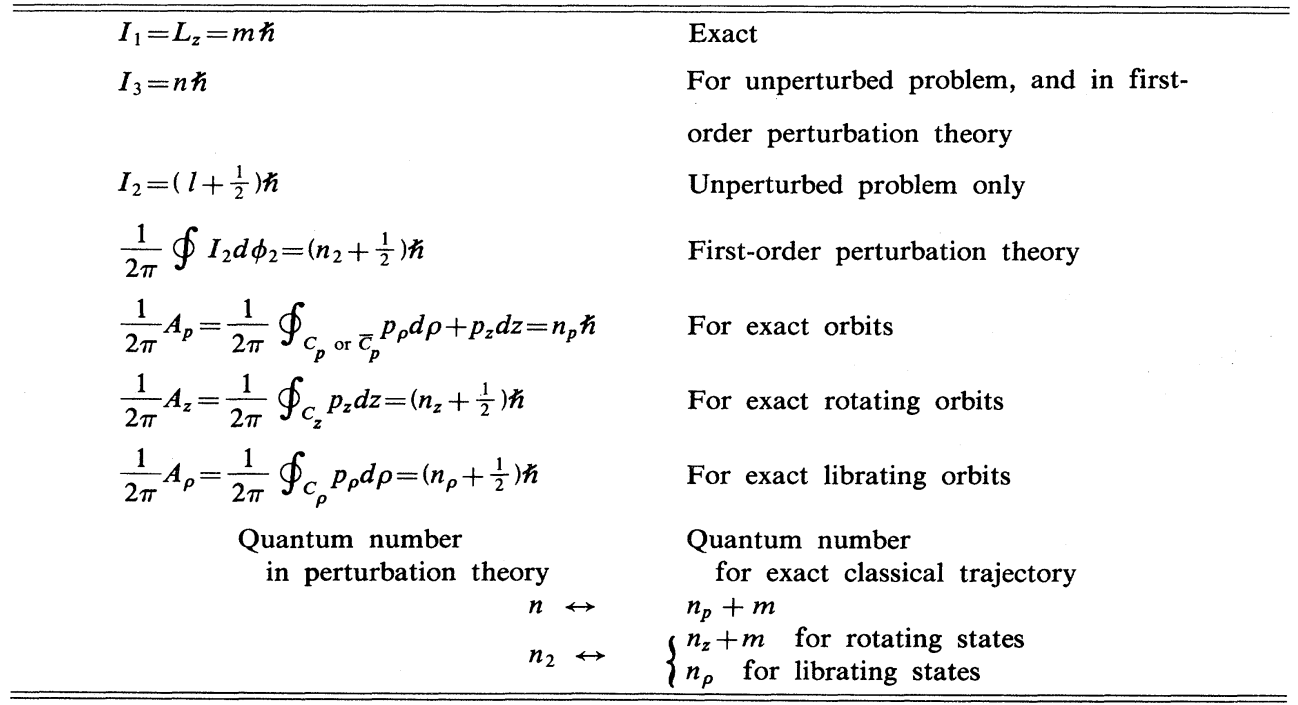




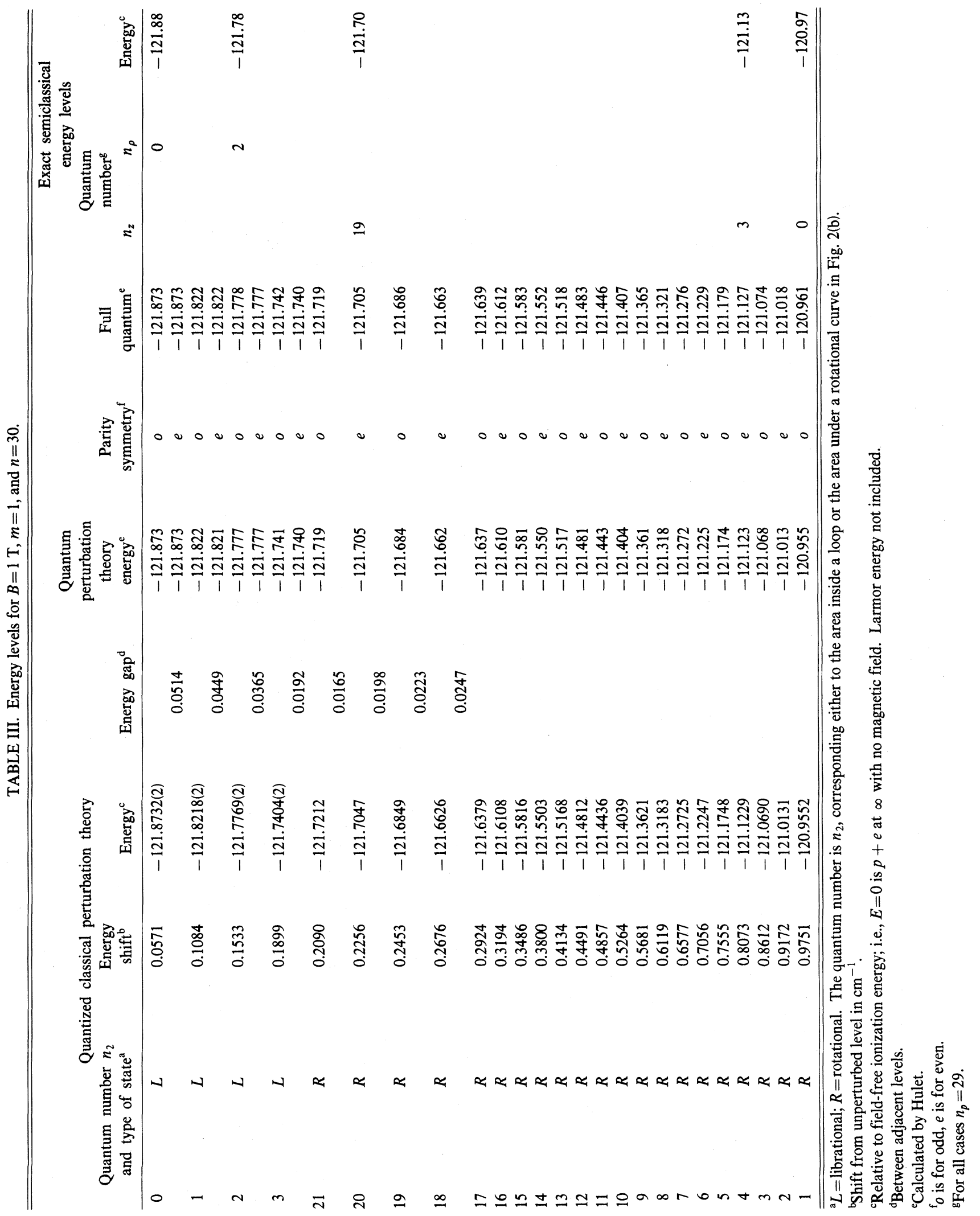




$$
n_{2}=n_{\rho} .
$$

These quantization conditions and the relationships between them are summarized in Table II.

\section{E. Energy spectrum}

Calculated energy levels for $B=1 \mathrm{~T}, n=30$, and $m=1$ are presented in Table III. The first set of columns shows the result of quantized classical perturbation theory, Eq. (45). The numerical precision ${ }^{13}$ of this calculation is such that the error in the last digit should not be more than \pm 2 . The next column shows corresponding results of quantum perturbation theory; these energies were obtained by expanding the wave function in a hydrogenic basis, and diagonalizing the Hamiltonian matrix within the 29-fold degenerate manifold of states having $m=1$ and $n=30$. (The matrix was first divided into two uncoupled parts, a $15 \times 15$ block of odd-parity states and a $14 \times 14$ block of even-parity states; then eigenvalues within each block were calculated numerically.) This calculation, as well as the "full quantum" calculation, were kindly provided to us by Hulet.

Examining these results, one finds the following.

(i) At low energies there are four doubly degenerate librational levels, or eight such states. This is in agreement with Eqs. (43) and (44), which for $I_{1}=1$ and $I_{3}=30$ give, respectively, $N_{L}=7.87$ and 8.17 .

(ii) The largest energy shift $\Delta E$ for a librational level is $\sim 0.19 \mathrm{~cm}^{-1}$, consistent with (19), which gives $0.20 \mathrm{~cm}^{-1}$ as the boundary between librational and rotational levels.

(iii) The quantum calculation shows some evidence of a tiny splitting between nearly degenerate librational states, but the energy gap is in the sixth figure, and we are not certain of the precision of this calculation.

(iv) The smallest energy shift is $0.0571 \mathrm{~cm}^{-1}$, and the largest is $0.9751 \mathrm{~cm}^{-1}$. These are consistent with the bounds predicted by (42), which are, respectively, 0.0291 and $1.004 \mathrm{~cm}^{-1}$.

(v) The smallest energy gap between adjacent levels is the one between $20 R$ and $21 R$, which are the two rotational levels lying closest to the separatrix.

(vi) To within the precision of these calculations, quantum perturbation theory and quantized classical perturbation theory are in essentially exact agreement with each other. The largest discrepancy between the two occurs for state $21 R$, and this might be associated with quantum effects related to the classical separatrix. ${ }^{14}$

An "exact" full quantum calculation was also carried out. In this case the expansion of the wave function included all hydrogenic states having $m=1$ lying between $n=27$ and 34. Results of this calculation are also shown in Table III. These exact energies are essentially identical to those obtained by quantum perturbation theory except in the following respects.

(i) The apparent splitting between nearly degenerate librational levels might be very slightly increased, but it is still very small.

(ii) The energies of the highest levels are slightly decreased. This represents the usual sort of higher-order correction to first-order perturbation theory. The firstorder energy correction is just $\lambda H_{1}$ averaged over an unperturbed orbit or quantum state. In second order, the trajectory or wave function adjusts to the perturbation,

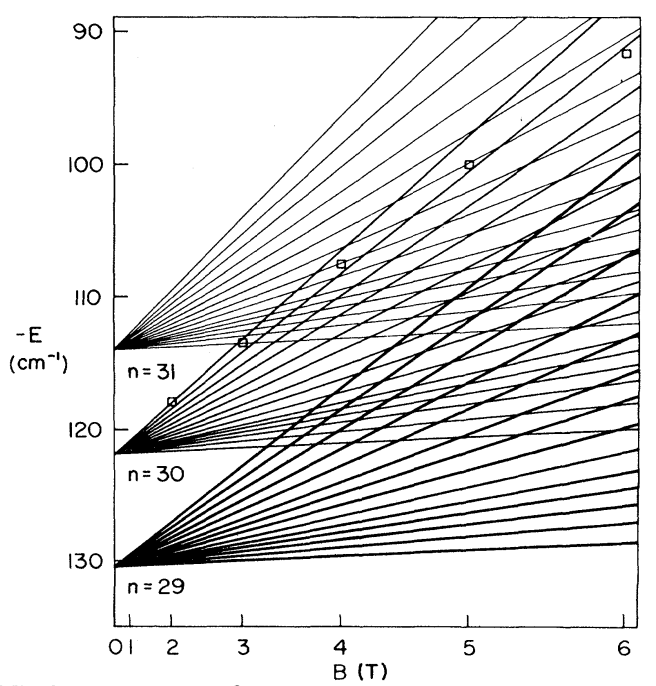

FIG. 8. Spectrum of energy levels for $m=1, n=29,30$, and 31. Solid lines are results of perturbation theory. Points are exact semiclassical eigenvalues for the highest-energy state in the $n=30$ manifold.

shifting away from regions in which $\lambda H_{1}$ is large.

We also calculated energy levels using exact numerically computed trajectories and action-variable quantization, as discussed in Sec. V A. For $B=1 \mathrm{~T}$, these calculations are quite time consuming, because Kepler ellipses precess very slowly, and it takes many cycles to obtain the data of Fig. 7. We obtained energies for only a few representative states. Table III shows agreement between this calculation and the full quantum calculation.

Energy levels as a function of magnetic field strength are shown in Fig. 8. These are in very good agreement with calculations and experiments reported in Ref. 5.

\section{CONCLUSION}

Using a combination of perturbation theory and exact trajectory calculations, we have studied classical orbits of an electron in a high Rydberg state in an external magnetic field. By quantizing the resulting action variables we have calculated the spectrum of discrete energy levels. Our results are fully consistent with quantum calculations made for us by Hulet, which in turn are consistent with experimental measurements made at MIT. Our semiclassical pictures provide a way of interpreting such experiments.

\section{ACKNOWLEDGMENTS}

This work was supported by grants from the National Science Foundation (J.B.D.), from the College of William and Mary (S.K.K.), and from the U.S. Department of Energy (Contract No. W-7405-eng-26 with Union Carbide Corporation) (D.W.N.). We thank R. Hulet for the quantum calculations listed in Table III.

\section{APPENDIX A: EVALUATION OF THE EFFECTIVE HAMILTONIAN}

Equation (11) tells us that we have to average $\lambda H_{1}=\lambda\left(x^{2}+y^{2}\right)$ over time on a Kepler ellipse. The cal- 
culation can be done in a number of ways, most of which require some pages of analysis. The cleanest derivation we found is given below.

Define

$$
\bar{H}_{i j}=\frac{1}{\tau} \oint x_{i} x_{j} d t,
$$

where $x_{i}=x, y, z$ for $i=1,2,3$ and the integral is performed on a Kepler ellipse. The nine components $\bar{H}_{i j}$ form a tensor, which transforms under rotations of coordinates as

$$
\underline{\bar{H}}^{\prime \prime}=\underline{A} \underline{\bar{H}} \underline{A}^{\dagger}
$$

if components $\underline{x}^{\prime \prime}$ in rotated coordinates are related to components $\underline{x}$ in the original system by

$$
\underline{x}^{\prime \prime}=\underline{A} \underline{x} .
$$

Furthermore,

$$
\lambda \bar{H}_{1}=\lambda\left(\bar{H}_{x x}+\bar{H}_{y y}\right) .
$$

Consider the following sequence of coordinate transformations: (i) rotate by $\phi_{1}$ about the $z$ axis, (ii) rotate by $i$ about the new $x$ axis, and (iii) rotate by $\phi_{2}$ about the resulting $z$ axis. Then in the rotated coordinate system, the positive $z^{\prime \prime}$ axis coincides with the angular momentum vector, so the orbit lies in the $x^{\prime \prime} y^{\prime \prime}$ plane, and the positive $x^{\prime \prime}$ axis passes through the perihelion. It immediately follows from symmetry that

$$
\underline{\bar{H}}^{\prime \prime}=\left[\begin{array}{ccc}
\bar{H}_{x^{\prime \prime} x^{\prime \prime}} & 0 & 0 \\
0 & \bar{H}_{y^{\prime \prime} y^{\prime \prime}} & 0 \\
0 & 0 & 0
\end{array}\right]
$$

and we can calculate $\underline{\bar{H}}$ using the reverse transformations.

Rotation matrices for steps (ii) and (iii) are, respectively,

$$
\begin{aligned}
& \underline{A}_{i i}=\left[\begin{array}{ccc}
1 & 0 & 0 \\
0 & \cos i & \sin i \\
0 & -\sin i & \cos i
\end{array}\right], \\
& \underline{A}_{i i i}=\left[\begin{array}{ccc}
\cos \phi_{2} & \sin \phi_{2} & 0 \\
-\sin \phi_{2} & \cos \phi_{2} & 0 \\
0 & 0 & 1
\end{array}\right] .
\end{aligned}
$$

Multiplying out the matrices for the reverse transformations

$$
\underline{\bar{H}}=\underline{A}_{i i}^{\dagger} \underline{A}_{i i i}^{\dagger} \underline{\bar{H}}^{\prime \prime} \underline{A}_{i i i} \underline{A}_{i i}
$$

a moment's calculation gives

$$
\begin{aligned}
\lambda \bar{H}_{1}=\lambda & {\left[\bar{H}_{x^{\prime \prime} x^{\prime \prime}}\left(\cos ^{2} \phi_{2}+\cos ^{2} i \sin ^{2} \phi_{2}\right)\right.} \\
& \left.+\bar{H}_{y^{\prime \prime} y^{\prime \prime}}\left(\sin ^{2} \phi_{2}+\cos ^{2} i \cos ^{2} \phi_{2}\right)\right] .
\end{aligned}
$$

Rotation (i) and its inverse leave $\bar{H}_{1}$ invariant. Defining

$$
\bar{H}_{r r}=\bar{H}_{x^{\prime \prime} x^{\prime \prime}}+\bar{H}_{y^{\prime \prime} y^{\prime \prime}},
$$

and using

$$
\begin{aligned}
& \cos ^{2} \phi_{2}=\frac{1}{2}\left(1-\cos 2 \phi_{2}\right), \\
& \sin ^{2} \phi_{2}=\frac{1}{2}\left(1+\cos 2 \phi_{2}\right),
\end{aligned}
$$

we obtain

$$
\begin{aligned}
\lambda \bar{H}_{1}=\lambda[ & \bar{H}_{r r} \frac{1}{2}\left(1+\cos ^{2} i\right) \\
& \left.+\left(\bar{H}_{x^{\prime \prime} x^{\prime \prime}}-\frac{1}{2} \bar{H}_{r r}\right)\left(1-\cos ^{2} i\right) \cos ^{2} 2 \phi_{2}\right] .
\end{aligned}
$$

The integrals $\bar{H}_{r r}$ and $\bar{H}_{x^{\prime \prime} x^{\prime \prime}}$ are now most easily calculated using the so-called eccentric anomaly $\psi$ as an auxiliary variable,

$$
\bar{H}_{r r}=\frac{1}{\tau} \oint\left[\left(x^{\prime \prime}\right)^{2}+\left(y^{\prime \prime}\right)^{2}\right] d t=\frac{1}{\tau} \oint r^{2} d t
$$

since $z^{\prime \prime}=0$ on the orbit. Using Eqs. (3-69) and (3-76) of Goldstein's second edition, ${ }^{8}$

$$
r=a(1-\cos \psi), \frac{d t}{d \psi}=\frac{\tau}{2 \pi}(1-\cos \psi),
$$

we obtain

$$
\bar{H}_{r r}=\frac{a^{2}}{2 \pi} \int_{0}^{2 \pi}(1-e \cos \psi)^{3} d \psi .
$$

Only terms with even powers of $\psi$ contribute, and

$$
\bar{H}_{r r}=a^{2}\left(1+\frac{3}{2} e^{2}\right) .
$$

For $\bar{H}_{x^{\prime \prime} x^{\prime \prime}}$ we note that

$$
x^{\prime \prime}=r \cos \chi
$$

and that the geometrical angle $\chi$ between the perihelion and the instantaneous position of the particle is related to the eccentric anomaly $\psi$ by Goldstein's Eq. (3-77),

$$
\cos \chi=(\cos \psi-e) /(1-e \cos \psi),
$$

so

$$
\begin{aligned}
\bar{H}_{x^{\prime \prime} x^{\prime \prime}} & =\frac{1}{\tau} \oint r^{2} \cos ^{2} \chi d t \\
& =\frac{a^{2}}{2 \pi} \int_{0}^{2 \pi}(1-e \cos \psi)(\cos \psi-e)^{2} d \psi \\
& =a^{2}\left(2 e^{2}+\frac{1}{2}\right)
\end{aligned}
$$

Combining (A8), (A10), and (A11), we obtain

$$
\begin{aligned}
\lambda \bar{H}_{1}=\lambda a^{2}[ & \left(1+\frac{3}{2} e^{2}\right) \frac{1}{2}\left(1+\cos ^{2} i\right) \\
& \left.+\frac{5}{2} e^{2} \frac{1}{2}\left(1-\cos ^{2} i\right) \cos ^{2} 2 \phi_{2}\right] .
\end{aligned}
$$

This can be reexpressed in terms of action-angle variables using formulas in Table I, and the result is Eq. (12).

\section{APPENDIX B: EQUATIONS OF MOTION}

For reference we list here the equations of motion for $\phi_{1}, \phi_{2}$, and $I_{2}$, derived from Hamilton's canonical equations using Eq. (12),

$$
\left\langle\frac{d \phi_{1}}{d t}\right\rangle=\lambda \frac{\partial \bar{H}_{1}}{\partial I_{1}}=\frac{\lambda I_{3}^{2}}{2 \mu^{2} k^{2} I_{2}^{2}}\left[\left(5 I_{3}^{2}-3 I_{2}^{2}\right) I_{1}-5 \cos 2 \phi_{2} I_{1}\left(I_{3}^{2}-I_{2}^{2}\right)\right],
$$




$$
\begin{aligned}
\left\langle\frac{d \phi_{2}}{d t}\right\rangle & =\lambda \frac{\partial \bar{H}_{1}}{\partial I_{2}}=\frac{-\lambda I_{3}^{2}}{2 \mu^{2} k^{2} I_{2}^{3}}\left[\left(5 I_{1}^{2} I_{3}^{2}+3 I_{2}^{4}\right)-5 \cos 2 \phi_{2}\left(I_{1}^{2} I_{3}^{2}-I_{2}^{4}\right)\right], \\
\left\langle\frac{d I_{2}}{d t}\right\rangle & =-\lambda \frac{\partial \bar{H}_{1}}{\partial \phi_{2}}=\frac{+\lambda I_{3}^{2}}{2 \mu^{2} k^{2} I_{2}^{2}}\left[5\left(I_{2}^{2}-I_{1}^{2}\right)\left(I_{3}^{2}-I_{2}^{2}\right) \sin 2 \phi_{2}\right] .
\end{aligned}
$$

The angular brackets $\langle d x / d t\rangle$ mean that the time derivative is averaged over one Kepler cycle.

\section{APPENDIX C: NUMBER OF LIBRATIONAL STATES}

To compute the area inside the two $U$-shaped separatrices, we find the area inside half of one of them, and multiply by 4 ,

$$
2 \pi N_{L}=4\left[I_{3}\left[\phi_{2}^{T}-\frac{\pi}{2}\right]-\int_{\pi / 2}^{\phi_{2}^{T}} I_{2}\left(\phi_{2}\right) d \phi_{2}\right],
$$

where $I_{2}\left(\phi_{2}\right)$ refers to the right-hand half of the $U$. The integral in Eq. (C1) is most easily evaluated using $I_{2}$ as the independent variable

$$
\int_{\pi / 2}^{\phi_{2}^{T}} I_{2}\left(\phi_{2}\right) d \phi_{2}=\int_{I_{2}^{U}}^{I_{3}} I_{2} \frac{d \phi_{2}}{d I_{2}} d I_{2}
$$

$$
\frac{d \phi_{2}}{d I_{2}}=\frac{2 I_{1}^{2}}{\left(I_{2}^{2}-I_{1}^{2}\right)\left(I_{2}^{2}-5 I_{1}^{2}\right)^{1 / 2}}
$$

and the substitution

$$
u=\left(I_{2}^{2}-5 I_{1}^{2}\right)^{1 / 2}
$$

reduces $(\mathrm{C} 2)$ to

$$
2 I_{1}^{2} \int_{0}^{\left(I_{3}^{2}-5 I_{1}^{2}\right)^{1 / 2}} \frac{d u}{\left(u^{2}+4 I_{1}^{2}\right)}=I_{1} \cos ^{-1}\left(\frac{2 I_{1}}{\left(I_{3}^{2}-I_{1}^{2}\right)^{1 / 2}}\right) \text {. }
$$

Therefore

$$
N_{L}=\frac{2}{\pi}\left[I_{3}\left(\phi_{2}^{T}-\frac{\pi}{2}\right)-I_{1} \cos ^{-1}\left(\frac{2 I_{1}}{\left(I_{3}^{2}-I_{1}^{2}\right)^{1 / 2}}\right)\right] .
$$

From Eq. (21), we have on the separatrix

${ }^{1}$ M. Born, The Mechanics of the Atom (Ungar, New York, 1960). ${ }^{2}$ E. U. Condon and G. H. Shortley, Theory of Atomic Spectra (Cambridge University Press, Cambridge, England, 1963).

${ }^{3}$ L. I. Schiff and H. Snyder, Phys. Rev. 55, 59 (1939); A. F. Starace, J. Phys. B 6 , 585 (1973); A. F. Starace and G. L. Webster, Phys. Rev. A 19, 1629 (1979); J. M. Wadehra, Astrophys. J. 226, 372 (1978); V. Canuto and D. C. Kelly, Astrophys. Space Sci. 17, 277 (1972); J. J. Labarthe, J. Phys. B 14, 1467 (1981); C. W. Clarke, Phys. Rev. A 24, 605 (1981); A. G. Zhilich and B. S. Monozon, Fiz. Tverd. Tela (Leningrad) $\underline{8}$, 3559 (1966) [Sov. Phys. Solid State $\underline{8}, 2846$ (1967)]; A. R. P. Rau, Phys. Rev. A 16, 613 (1977); H. Hasegawa and R. E. Howard, J. Phys. Chem. Solids 21, 179 (1961); R. J. Elliott and R. Loudon, ibid. 15, 196 (1960); D. R. Herrick, Phys. Rev. A 26, 323 (1982); R. Cohen, J. Lodenquai, and M. Ruderman, Phys. Rev. Lett. 25, 467 (1970); M. Ruderman, ibid. 27, 1306 (1971); E. G. Flowers, J.-F. Lee, M. A. Ruderman, P. G. Sutherland, W. Hillebrandt, and E. Müller, Astrophys. J. 215, 291 (1977); R. H. Garstang, Rep. Prog. Phys. 40, 105 (1977); J. C. Gay, in Proceedings of NATO Advanced Study Institute on Photophysics and Photochemistry in the Vacuum Ultraviolet (in press). An analysis quite similar to that given here, but carried out less completely, is given by $\mathrm{E}$. A. Solov'ev, Zh. Eksp. Teor. Fiz. Pis'ma Red 34, 278 (1981) [JETP Lett. 34, 265 (1981)].

${ }^{4}$ I. C. Percival, J. Phys. B 6, L229 (1973); Adv. Chem. Phys. 36, 1 (1977); M. V. Berry, Philos. Trans. R. Soc. London, Ser. A 287, 237 (1977); J. Phys. A 10, 2083 (1977).

${ }_{5}^{5}$. L. Zimmerman, J. C. Castro, and D. Kleppner, Phys. Rev. Lett. 40, 1083 (1978); J. C. Castro, M. L. Zimmerman, R. G.
Hulet, D. Kleppner, and R. R. Freeman, ibid. 45, 1780 (1980); D. Kleppner, M. G. Littman, and M. L. Zimmerman, Sci. Am. 244, 130 (1981).

${ }^{6}$ Note that since we took the nucleus to be infinitely massive, we are not distinguishing between the rest mass of the electron and its reduced mass on an atom. The usual separation of center of mass and relative motion does not apply when the atom is in a magnetic field.

${ }^{7}$ There is an interesting problem in celestial mechanics that has the same Hamiltonian. Suppose we have a planet moving around a fixed star, and star and planet are embedded in a uniform constant density cylinder of dust centered at the star. Imagine that the dust cloud causes no significant friction on the planet, but it generates a gravitational force on the planet, and a corresponding potential energy which is proportional to $\rho^{2}$. The motion of this planet will be the same as the classical motion of an electron in a magnetic field, if the electron is viewed from a frame of reference that is precessing at the Larmor frequency.

${ }^{8}$ H. Goldstein, Classical Mechanics, 2nd ed. (Addison-Wesley, Reading, Mass., 1980).

${ }^{9}$ All numbered equations in this section hold for all values of $I_{1}$, not just $\left|I_{1}\right|=1$.

${ }^{10}$ A. Einstein, Verh. Dtsch. Phys. Ges. 19, 82 (1917); R. A. Marcus, Discuss. Faraday Soc. $\underline{55}, 34$ (1973); D. W. Noid and R. A. Marcus, J. Chem. Phys. 62, 2119 (1975); V. P. Maslov and M. V. Fedoriuk, Semiclassical Approximation in Quantum Mechanics (Reidel, Boston, 1981).

${ }^{11}$ The quantity $p_{z} d z$ is not invariant under canonical transformations, and $\int p_{z} d z$ on an arbitrary path is not an action 
variable. The correct prescription for an arbitrary loop is Eq. (32), and since we take $\rho=$ const, $d \rho=0$ and the action integral reduces to (33).

${ }^{12}$ T. Poston and I. Stewart, Catastrophe Theory and its Applications (Pitman, London, 1978); M. V. Berry, Adv. Phys. 25, 1 (1976); J. N. L. Connor, Mol. Phys. 31, 33 (1976).

${ }^{13}$ Here we are drawing the usual distinction between precision and accuracy. Our numerical techniques should give almost seven-figure precision for the energy levels, but perturbation theory is accurate in this case only to about six figures (less for the highest levels). Also, some error arises from taking $\mu=1$, which we used for consistency with the calculations made for us by Hulet.

${ }^{14}$ In one-dimensional quantum systems such as a pendulum, tunneling is important just below the separatrix, while just above the separatrix the potential-energy barrier causes some reflection. One expects that similar phenomena will occur here. 